\title{
A recognition masking study of consonant processing
}

\author{
CATHERINE G. WOLF \\ Research Laboratory of Electronics, Massachusetts Institute of Technology \\ Cambridge, Massachusetts 02199
}

\begin{abstract}
The processing of consonants was investigated in a series of experiments using a recognition masking paradigm. Experiment I investigated the effects of target duration, interstimulus interval, forward vs. backward masking, and the phonetic feature composition of the target and mask on accuracy of target identification. Experiment II assessed consonant processing when the target and mask were presented dichotically in order to separate central and peripheral components of consonant masking. Experiment III investigated the effects of mask duration on consonant processing. Substantial masking was found in backward and forward diotic and dichotic conditions. Evidence for target-mask interaction at the level of phonetic features was also found. The results of the experiments indicated that the processes leading to phonetic categorization of a consonant may continue beyond stimulus offset and may be interfered with by the presentation of another consonant before or after the target. The experiments also suggested the existence of two loci of target-mask interaction: a peripheral locus and a central locus.
\end{abstract}

A primary concern of research on the perception of speech is the problem of how acoustic information is converted into abstract phonetic information. Given the acoustic signal as input, and the identification of a phonetic segment as the desired output, researchers in the field of speech perception have attempted to describe the events intervening between input and output in terms of a series of discrete stages (Liberman, 1970; Morton \& Broadbent, 1967; Stevens \& Halle, 1967; Stevens \& House, 1972; Studdert-Kennedy, 1970). The present study investigated the stages of speech processing using a paradigm which has proved useful in the investigation of nonspeech information processing: the recognition masking paradigm (cf. Massaro, 1972a; Turvey, 1973).

In recognition masking, one of several target stimuli is presented before or after a masking stimulus. The subject's task is to identify the target. When the target precedes the mask, the paradigm is referred to as backward masking; when the target follows the mask, the paradigm is referred to as forward masking. Masking is said to occur when accuracy of target identification is impaired by presentation of the mask. By assessing the effects of a number of temporal and stimulus variables on target

The research reported in this article was conducted in partial fultillment of the requirements for the $\mathrm{PhD}$ degree in psychology at Brown University (Wolf. 1974). I am indebted to my advisor. Peter D. Eimas, whose guidance and critical comments at all stages, from the designing of the experiments to the preparation of the manuscript. were indispensable. I would like also to thank Franklin $S$. Cooper for his generosity in making the facilities of Haskins Laboratories available, and Kenneth $\mathbf{N}$. Stevens for his comments on an earlier draft of this paper. This research was supported in part by an NIMH predoctoral fellowship. F01 51-085, to the author and in part by Grant HD 05331 from the National Institute of Child Health and Human Development to P. D. Eimas. Some of the data reported herein have been previously described in a paper presented at the 1974 meeting of the Eastern Psychological Association. identification, researchers have attempted to map the stages involved in the identification of auditory and visual stimuli.

On the basis of recognition masking studies using tones as targets and masks, Massaro (1972a, b) has distinguished two stages of auditory processing: a first stage, during which an initial, transient, uncategorized stimulus representation is formed (the preperceptual auditory image), and a second stage, during which information is read out into a categorized form. In the case of brief stimuli, processing at these stages may continue beyond stimulus off'set.

Massaro has proposed that the mask in the backward masking paradigm impairs accuracy of target identitication because the onset of the mask disrupts the readout of the target preperceptual auditory image into a categorized form. Consequently, when masking occurs, information about the target is obliterated. Support for Massaro's proposal comes primarily from the findings that: (1) the similarity between the frequency (hertz) of the target and mask tones does not influence the effectiveness of the masking tone (Massaro, 1971); (2) little or no fonward masking is found over a range of interstimulus intervals which produce backward masking (Massaro, 1970, 1973). If the mask does not obliterate the target representation, but rather, interacts with it. one might expect the effectiveness of a particular mask to depend on target-mask similarity. Similarly, if masking involves an interaction between the target and mask, one might expect forward. as well as backward. masking to occur.

In the last several years, the processing of speech sounds has been investigated using a variant of the standard recognition masking paradigm. In these studies. the two stimuli overlap temporally and the 
interval between the onset of the first and onset of the second stimulus is varied. This research has found evidence for forward as well as backward masking for consonant-vowel (CV) targets and masks (StuddertKennedy. Shankweiler, \& Shulman, 1970). In fact, Studdert-Kennedy et al. (1970) found that monotic presentation of the target and mask resulted in greater forward than backward masking. ${ }^{1}$ With dichotic presentation. backward masking was more severe than forward masking.

Other dichotic studies have found that target-mask similarity may affect accuracy of target identification. Darwin (1971) assessed accuracy of identification for a set of CVs when the mask was either another CV with the same vowel, the steady-state vowel, or a nonspeech sound. In the backward masking condition, the $C V$ was the most effective mask and the nonspeech sound was the least effective. Similarly, Pisoni and McNabb (1974) found that masking was most severe for $\mathrm{CV}$ targets and masks which shared the same vowel, and decreased as the similarity between the target and mask vowel decreased. When the target and mask syllables had the same vowel and the consonants differed by only one or two phonetic features, accuracy of target identification was greater for target and mask consonants differing by one phonetic feature than for those differing by two features (Pisoni \& McNabb, 1974). Similar results were found by McNeill and Repp (1973) for forward masking. Because the target and mask overlapped in these experiments, identification of the target may have required an initial separation of the target and mask signals. Large spectral differences between the target and mask, such as those produced by different vowels (Pisoni \& McNabb, 1974) or different classes of sounds (Darwin, 1971), may have facilitated the separation of the target and mask at a relatively early stage of consonant processing and, thus, reduced masking.

The effect of the number of consonant features distinguishing a target and mask on the spectral distinctiveness of that target and mask is relatively small compared to the effect on spectral distinctiveness of the number of vowel features distinguishing a target and mask. Consequently, targets and masks differing by two consonant features may not be more efficiently separated than targets and masks differing by one feature. In such cases, the effect of target-mask feature contrasts may arise at a higher level of processing: the level of phonetic feature analysis.

It is suggested that the feature contrast effect is caused by competition between the target and mask for the same feature analyzers at the level of phonetic feature extraction. Such competition may result in a loss of target feature information and the subsequent replacement of target feature values by the corresponding mask feature values. When the target and mask differ by only one feature, replacement of target features by mask features is less likely to produce a change in the phonetic feature composition of the target representation than when the target and mask differ by two features. Consequently, target identification is more accurate when the target and mask differ by one. rather than two, phonetic features. ${ }^{2}$ These ideas are explored further in the present experiments.

The forward masking and the apparent target-mask similarity effect observed in these studies of dichotic overlap masking suggest that the mask did not simply obliterate the target, but, rather. interacted with the target. Because it is possible that the differences between the findings for speech and nonspeech stimuli were due to differences between the paradigms used in the investigation of these stimuli, the present studies were intended to investigate consonant processing using the standard backward and forward recognition masking paradigms. The results of some studies of consonant processing using the standard backward masking paradigm by Pisoni (Note 1) and, recently, Massaro (1974) indicate that recognition masking paradigms may be useful in napping the course of consonant processing.

Experiment $I$ investigated the effects of the interstimulus interval (the interval between the offset of the first stimulus and onset of the second. ISI). target duration, and the phonetic feature composition of the target and mask on consonant processing under diotic listening conditions. Experiment II was undertaken to determine whether the findings of Experiment I were attributable to central as opposed to peripheral processes in speech perception. Central processes are detined as those operations that occur after dichotic input to the two ears reaches a conmon processor. Peripheral processes (also referred to as transmission line processes) are defined as those operations occurring prior to central operations in the sequence of speech processing. Thus, diotic target-mask presentation. employed in Experiment I, permits both peripheral and central interactions, whereas dichotic presentation permits central interactions only. The target and mask were, therefore, presented dichotically in Experiment II in the expectation that a comparison of the results of Experiments I and II would provide information concerning the nature of central and peripheral processes in the perception of speech. The findings of Experiments I and II will be discussed together in order to facilitate a comparison of their results.

\section{EXPERIMENT I}

\section{Method}

\section{Subjects}

Eleven male and female students at Brown University served as subjects. All were native speakers of American English with no 
known hearing impairment in either ear. Subjects were paid $\$ 10$ for their participation in the experiment.

\section{Stimuli}

The stimuli were three-formant synthetic speech sounds generated on the computer-controlled parallel resonance synthesizer at Haskins Laboratories, New Haven, Connecticut. The target set consisted of $[d, g, k]$, and the mask set consisted of $[b, p$, t]. The six stimuli were taken from the series constructed by Lisker and Abramson (1970) and modified for the purposes of this experiment. In order to reduce the possibility that processing of the target consonant would be completed during the vowel portion of the stimulus, the duration of this portion was reduced to the minimum judged necessary for the perception of the syllable as speech at the shortest target duration used. Target durations of 22, 44 , and $88 \mathrm{msec}$ were employed. The $44-$ and $88-\mathrm{msec}$ target durations were produced by expanding each $2 \cdot \mathrm{msec}$ segment of the $22-\mathrm{msec}$ targets equally. This method of manipulating target duration kept the relative duration of each segment of the stimulus constant across target durations. Target duration was manipulated in this way rather than by extending the steady-state vowel portion of the stimulus in order to assess the effects on accuracy of target identification of the duration of the interval over which critical target information occurred. The mask duration was $88 \mathrm{msec}$. The shortened vowel portion of the stimuli was heard as [ $[\ni]$ rather than [a], as in the original stimuli. A spectrogram of an 88-msec [d] target is shown in Figure 1.

The phonetic feature values of the target and mask stimuli for the voicing and place of articulation features are shown in Table 1 . The stimuli have identiçal values on all other phonetic features. As Table 1 indicates, the target and mask sets cannot be distinguished from each other on the basis of a consistent set of phonetic features.

\section{Design and Procedure}

Construction of the stimulus tapes. All stimulus tapes were prepared using the Pulse Code Modulation system at Haskins Laboratories. Two basic types of test orders were made: masked identification and unmasked identification. A masked identification test order consisted of each of the three target set members presented with each of the three mask set members at each of the seven ISIs $(0,10,20,40,80,120$, and $200 \mathrm{msec})$. Separate test orders were made for the backward and forward masking conditions, and, within a test order, target duration was constant. Two different random orders were made for each combination of target duration and type of masking paradigm (backward and forward).

For the backward masking condition, the sequence of events on each trial was as follows: (1) $1,000-\mathrm{Hz}$ warning tone $(250 \mathrm{msec})$ recorded on both tracks of the tape, (2) 1-sec silent foreperiod, (3) the target recorded on one track, (4) variable ISI, (5) the mask recorded on the other track, (6) 3-sec intertrial interval (ITI), during which the subject was to identify the target. The sequence of events on each trial for the forward masking condition was identical, with the exception that the target occurred in Interval 5 and the mask in Interval 3.

The unmasked identification test orders consisted of the targets presented without masks. Two test orders containing 20 presentations of each of the three target set members were made for each target duration. A 3-sec ITI intervened between successive stimuli.

Testing procedure. The experiment was administered in three sessions of about $75 \mathrm{~min}$ each. At the beginning and end of each

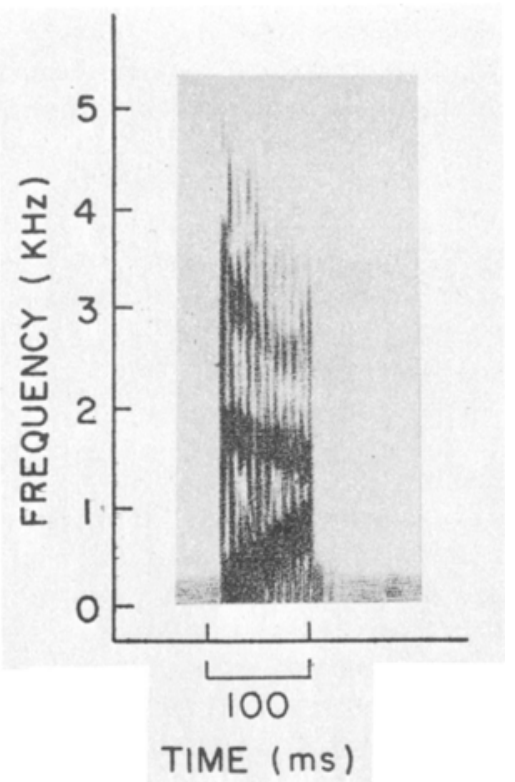

Figure 1. A spectrogram of the 88-msec target [d] is shown.

session, an unmasked identification test order was presented at each target duration. After the initial unmasked identification test orders, the masked identification test orders were presented. The forward and backward masking test orders were given in an alternating sequence, and each of the three target durations was presented once before any were repeated. For both the unmasked and masked identification tasks, subjects recorded their answers on an answer sheet using the letters "D," "G," and "K." Subjects were instructed to guess when they were unsure. No feedback was given concerning correctness for either the masked or unmasked identification tasks in order to insure that a subject's responses reflected his own perception of the stimuli and not an experimenter-imposed labeling.

At the beginning of the first session, a demonstration tape containing one presentation of each of the three targets at the 88-msec duration was played twice and the stimuli were identified by the experimenter. In addition, after the demonstration tape, a practice unmasked identification test order was presented for each target duration. Results from this practice run were not included in the data analysis.

Across the three sessions, subjects received 360 target presentations at each duration in the unmasked identification task. In the masked identification task, each combination of ISI by Target Duration by Masking Paradigm (backward or forward) was tested 36 times ( 3 target set members by 3 mask set members by 4 replications).

\section{Apparatus}

The tapes were played on a two-track Ampex recorder at the rate of $71 / 2 \mathrm{in} . / \mathrm{sec}$. The outputs from the two channels were mixed so that the tapes were heard diotically. Subjects listened to the tapes over Koss Pro 600AA stereo headphones.

Table 1

Phonetic Feature Values of the Targets and Masks on the Voicing and Place of Articulation Features

\begin{tabular}{lllllll}
\hline Feature & Target D & Target G & Target K & Mask B & Mask P & Mask T \\
\hline Voice & Voiced & Voiced & Voiceless & Voiced & Voiceless & Voiceless \\
Place & Alveolar & Velar & Velar & Bilabial & Bilabial & Alveolar \\
\hline
\end{tabular}




\section{Results \\ Unmasked Identification}

The mean percentage correct for target durations of 22,44 , and $88 \mathrm{msec}$ in the unmasked identification condition was 83,97 , and 95 , respectively. A one-way analysis of variance revealed the difference due to target duration to be statistically significant $[F(2,20)$ $=12.17, \mathrm{p}<.001] . \mathrm{A}$ post hoc analysis using Lindquist's (1953) critical difference indicated that the differences between the 22- and 44-msec and 22 and $88-\mathrm{msec}$ target durations were significant, $\mathrm{p}<.01$.

\section{Backward Masking}

Effects of ISI, target duration. Figure 2a presents the mean percentage correct as a function of ISI for the three target durations in the backward masking condition and the percentage correct for each target duration in the unmasked condition. Separate analyses of variance were performed for the backward and forward masking çonditions using number correct as the dependent variable. In each analysis,

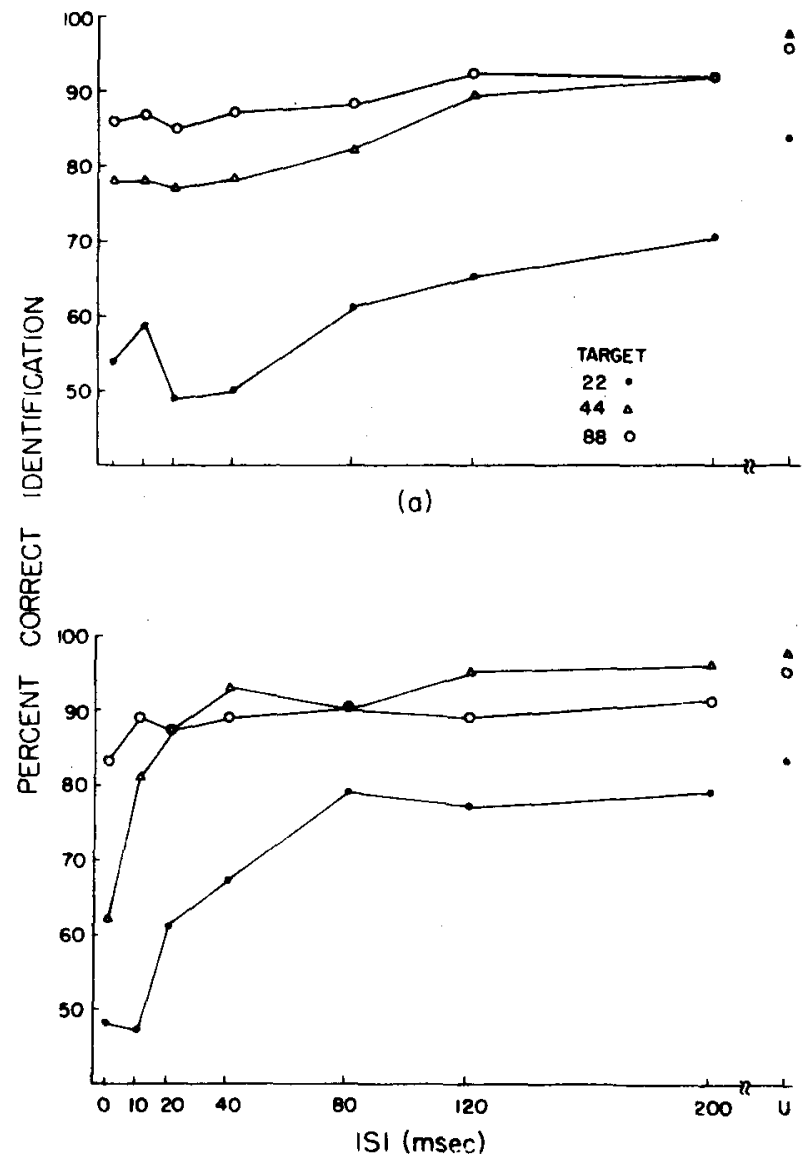

(b)

Figure 2. Mean percent correct identification $(N=11)$ is plotted as a function of ISI for target durations of $22 \mathrm{msec}$ (filled circles), 44 msec (triangles), and 88 msec (open circles) for the backward masking condition in (a) and the forward masking condition in (b), Experiment $I$. The unmasked level of performance for each target duration is indicated at the right by "U." accuracy was assessed as a function of the factors ISI and target duration. The analysis for the backward masking condition found significant effects for ISI $[F(6,60)=24.26, p<.001]$, duration $[F(2,20)$ $=50.40, \mathrm{p}<.001]$, and the ISI by Duration interaction $[\mathrm{F}(12,120)=2.44, \mathrm{p}<.01]$. The mean percentage correct was 58,82 , and 88 for target durations of 22,44 , and $88 \mathrm{msec}$, respectively. Post hoc comparisons between the means for the duration factor revealed significant differences in accuracy between the 22 and $44 \mathrm{msec}$ target duration means and the 22 and $88 \mathrm{msec}$ duration means. $\mathrm{p}<.01$.

The effect of ISI and the nature of the ISI by Duration interaction are illustrated in Figure $2 a$. For target durations of 44 and $88 \mathrm{msec}$, the functions are essentially flat up to an ISI of 20 to $40 \mathrm{msec}$ and then increase monotonically. The function for the $22-\mathrm{msec}$ duration, however, is nonmonotonic; accuracy is significantly lower at 20 - and $40-\mathrm{msec}$ ISI than at 10 and $80-\mathrm{msec}$ ISI $(\mathrm{p}<.01)$. Accuracy remains below the unmasked level at all ISIs for each target duration.

Effect of phonetic feature composition of target and mask. Evidence for an interaction between the target and mask on the level of phonetic features was assessed by comparing accuracy for target-mask pairs contrasting in one as opposed to two phonetic features (voicing or place of articulation alone vs. voicing and place together). If, as suggested above, target features may be lost and mask features may replace the lost target features in the target representation, accuracy of target identification should be greater for targets and masks differing by one feature than for those differing by two features. The rationale for this prediction is that replacement of target features by mask features is less likely to result in a change in the phonetic feature composition of the target representation (and, hence, result in an identification error) when the target and mask contrast in one feature than when they contrast in two features. For example, if the voicing feature value of the target $[\mathrm{g}]$ (voiced) is replaced by the corresponding feature value of the mask [b] (voiced), there is no change in the phonetic feature composition of the target representation. Feature replacement in this case does not result in an error. On the other hand, if replacement of the voicing feature occurs for $[\mathrm{g}]$ when the mask is [p] (voiceless), the phonetic feature composition of the target representation is changed and an error is predicted. In order to control for differences in the identifiability of the three targets, the effect of the number of phonetic feature contrasts between the target and mask on accuracy of target identification was assessed separately for each target and each duration. The six feature contrast predictions tested are presented in Table $2 .^{3} \mathrm{~A}$ prediction such as (1) should be understood: Accuracy will be greater for the target-mask pair [d]-[b] than for the pair $[\mathrm{d}]-[\mathrm{p}]$. 
Table 2

Percentage Correct in the Indicated Category for the Feature Contrast Predictions

\begin{tabular}{|c|c|c|c|c|c|c|c|c|c|c|c|c|c|c|c|}
\hline \multirow{3}{*}{\multicolumn{2}{|c|}{ Prediction }} & \multicolumn{6}{|c|}{ Experiment I } & \multicolumn{4}{|c|}{ Experiment 11} & \multicolumn{4}{|c|}{ Experiment III } \\
\hline & & \multirow{2}{*}{\multicolumn{3}{|c|}{$\begin{array}{c}\text { Backward } \\
\text { Target }\end{array}$}} & \multirow{2}{*}{\multicolumn{3}{|c|}{$\begin{array}{l}\text { Forward } \\
\text { Iration }\end{array}$}} & \multirow{2}{*}{\multicolumn{2}{|c|}{$\begin{array}{r}\text { Backward } \\
\text { Target }\end{array}$}} & \multicolumn{2}{|c|}{ Forward } & \multicolumn{2}{|c|}{ Dichotic } & \multicolumn{2}{|c|}{ Diotic } \\
\hline & & & & & & & & & & ratic & & & ask & atio & \\
\hline & & 22 & 44 & 88 & 22 & 44 & 88 & 22 & 44 & 22 & 44 & 22 & 88 & 22 & 88 \\
\hline 1 & $\mathrm{DB}>$ & 91 & 96 & 85 & 75 & 91 & 81 & 66 & 92 & 56 & 84 & 65 & 70 & 89 & 93 \\
\hline & & & $\begin{array}{l}95 \\
88\end{array}$ & 04 & & & & & & & 88 & & & & \\
\hline 2 & $\begin{array}{l}\text { DT }> \\
\text { DP }\end{array}$ & $\begin{array}{l}65 \\
85\end{array}$ & $\begin{array}{l}88 \\
95\end{array}$ & $\begin{array}{l}81 \\
84\end{array}$ & $\begin{array}{l}66 \\
71\end{array}$ & $\begin{array}{l}85 \\
89\end{array}$ & $\begin{array}{l}80 \\
86\end{array}$ & $\begin{array}{l}81 \\
74\end{array}$ & $\begin{array}{l}98 \\
91\end{array}$ & $\begin{array}{l}82 \\
67\end{array}$ & $\begin{array}{l}94 \\
88\end{array}$ & $\begin{array}{l}71 \\
67\end{array}$ & $\begin{array}{l}53 \\
65\end{array}$ & $\begin{array}{l}65 \\
7.4\end{array}$ & $\begin{array}{l}54 \\
84\end{array}$ \\
\hline 3 & $G B>$ & 58 & 86 & 89 & 71 & 88 & 92 & 74 & 96 & 78 & 95 & 69 & 64 & 70 & 60 \\
\hline & GP & 51 & 89 & 91 & 60 & 89 & 90 & 66 & 94 & 62 & 93 & 57 & 56 & 57 & 55 \\
\hline 4 & $\mathrm{~GB}>$ & 58 & 86 & 89 & 71 & 88 & 92 & 74 & 96 & 78 & 95 & 69 & 64 & 70 & 60 \\
\hline & GT & 45 & 91 & 87 & 54 & 87 & 88 & 47 & 86 & 50 & 88 & 47 & 30 & 50 & 29 \\
\hline 5 & $\mathrm{KP}>$ & 53 & 69 & 96 & 75 & 93 & 97 & 80 & 92 & 84 & 93 & 81 & 50 & 68 & 51 \\
\hline & KB & 21 & 49 & 83 & 47 & 66 & 84 & 56 & 69 & 53 & 72 & 44 & 27 & 57 & 15 \\
\hline 6 & $\mathrm{KT}>$ & 54 & 76 & 96 & 72 & 88 & 96 & 48 & 72 & 64 & 81 & 81 & 72 & 77 & 80 \\
\hline & KB & 21 & 49 & 83 & 47 & 66 & 84 & 56 & 69 & 53 & 72 & 44 & 27 & 57 & 15 \\
\hline
\end{tabular}

Table 2 also show's the percentage correct, averaged across subjects. for a target as a function of the mask, for each of the six predictions. Evaluation of these predictions at each target duration revealed that accuracy was greater for target-mask pairs contrasting in one feature than for those contrasting in two features in 12 out of $18(67 \%)$ instances ( $\mathrm{p}<.119$, sign test).

It can be argued, however, that target identification accuracy may vary as a function of the mask if the masking effect is based on an obliteration of all target information by the mask (see Footnote 2). The idea is that when masking occurs, subjects are left with no information about the target, and, consequently, their responses are based solely on the characteristics of the mask.

In order to establish that the feature contrast effect reported above was caused by an interaction of target and mask features, it is necessary to demonstrate that the mask does not disrupt target information in an all-or-none way. If the mask does disrupt target information in an all-or-none way, for any given mask. the pattern of error responses should be the same for all targets. If, on the other hand, the phonetic features of the target may be individually retained or lost. error responses should be determined by target phonetic features, as well as mask features.

According to the latter proposal, it is assumed that when target feature information is lost. the subject reports a member of the target set whose features match the remaining matrix of target features. Error responses should, thus, reflect the retained features. For example. [k] may be reported when the target $[\mathrm{g}]$ is presented if voicing information is lost, while place of articulation information is retained. For the target [d], however, an error response of [k] is predicted only if information about both voicing and place of articulation is lost. Although the probabilities of losing voicing and place information may not be independent (Repp, 1975), the probability of losing information about one feature is greater than the probability of losing information about two features.

The hypothesis of selective feature retention can be tested by examining the pattern of error responses for a particular mask as a function of the target (see Table 3). Since the total number of errors was not equal for the different targets, it was necessary to determine the percentage of the total errors which occurred in a given phonetic category for a particular target and compare this percentage for different targets with the same mask. For example, Prediction 1 of Table 3 compares the percentage of the total error responses which consist of $[k]$ for the targets $[\mathrm{g}]$ and $[\mathrm{d}]$ when the mask is [b]. A prediction such as (1) should be understood: Given the mask [b] and a target identification error, the percentage of $[\mathrm{k}]$ responses will be greater for the target $[\mathrm{g}]$ than for the target [d].

The six selective feature contrast predictions were evaluated for each target duration, averaged across subjects. Table 3 presents the percentage of errors in a particular category as a function of the target for each of the predictions. In two instances, there were no errors in the relevant category for either target, so the predictions cannot be evaluated. Of the remaining 16 cases. the predictions were supported in $13(81 \%)$ instances $(p<.011)$.

\section{Forward Masking}

Effects of ISI, target duration. An analysis of variance (ISI by Duration) was performed on the number correct, as in the backward masking condition. The following effects were found to be statistically signiticant: ISI $[F(6,60)=26.88]$. duration $[F(2.20)=23.05]$, and the ISI by Duration interaction $[F(12.120)=17.27]$ at the .001 level or better in each instance. The post hoc analysis of the duration factor revealed signiticant differences 


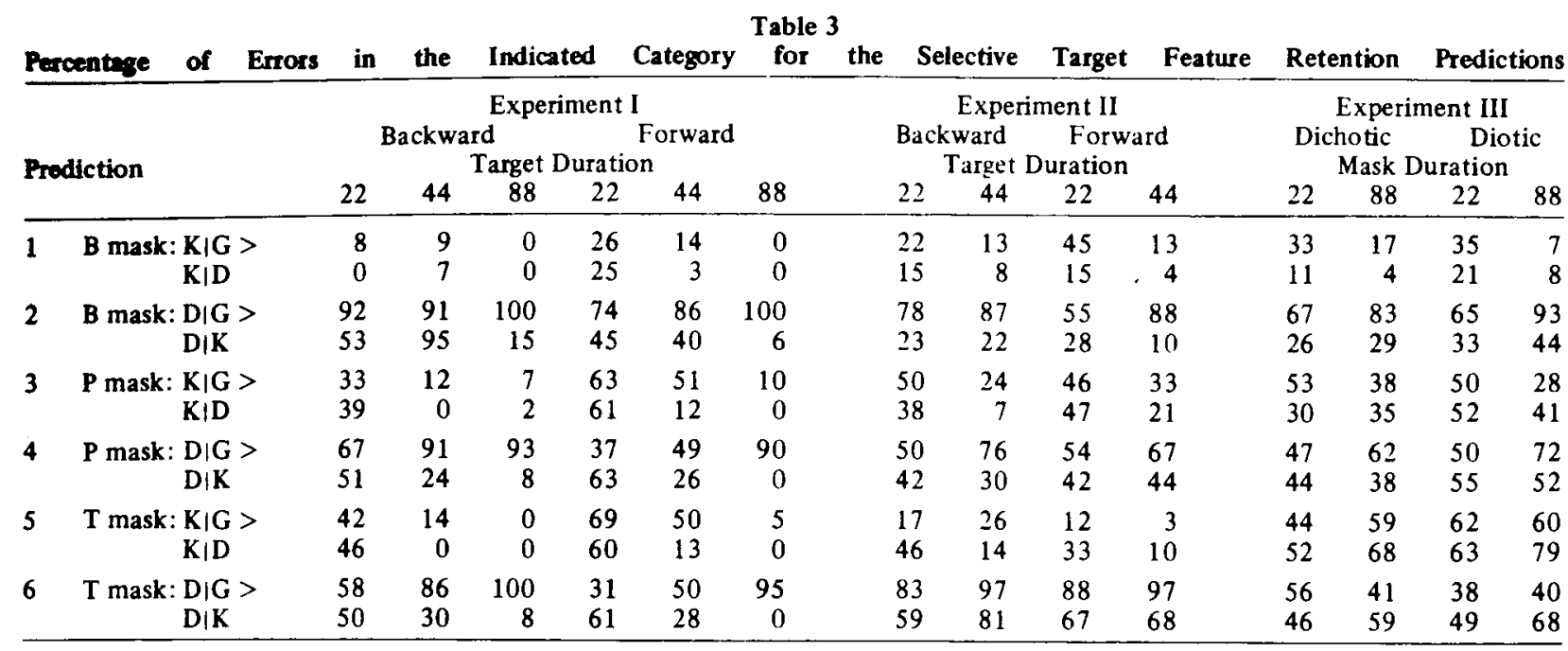

between the 22- and 44-msec durations and the 22 and $88-\mathrm{msec}$ durations, $\mathrm{p}<.01$. The mean percentage correct for the 22-, 44-, and $88-\mathrm{msec}$ durations was 66,86 , and 88 . The mean percentage correct is plotted as a function of ISI for each of the three durations in Figure $2 b$. It can be seen that accuracy increases monotonically with ISI, up to a point. for all target durations. The ISI by Duration interaction appears to be due to the lesser slope of the 88-msec function compared with the 22 - and $44-\mathrm{msec}$ functions at the shorter ISIs.

Effect of phonetic feature composition of target and mask. Thirteen of the $18(72 \%)$ phonetic feature contrast predictions were supported $(p<.048)$. Of the 17 cases in which the predictions concerning selective target feature retention could be evaluated (in one instance there were no errors in the relevant category for either target), the predictions were supported in $15(88 \%)$ instances $(p<.001)$. See Tables 2 and 3 for information about the individual feature contrast and selective target feature retention predictions, respectively.

\section{EXPERIMENT II}

\section{Method \\ Masked identification tapes were made for the 22 - and $44-\mathrm{msec}$ targets in which the targets and masks were recorded on separate channels and the channel receiving the target varied randomly from trial to trial. Unmasked identification tapes in which the channel receiving the target varied randomly were also made. \\ The apparatus used in testing the subjects was identical to that used in Experiment $\mathbf{I}$, with the exception that the outputs of the two channels were not externally mixed. Thus, the target and mask were presented dichotically.}

\section{Subjects}

Eight Brown University students sewed as subjects. All were right-handed native speakers of English and had no known hearing impairment in either ear.

\section{Design and Procedure}

The subjects were tested in three sessions of about $75 \mathrm{~min}$ each. The testing procedure was similar to that of Experiment I. In all. 180 stimuli of each target duration were presented to each ear in the unmasked identitication task, and there were 27 presentations of each ISI by Duration by Masking Paradigm by Ear Combination in the masked identification task.

\section{Unmasked Identification \\ Results}

An analysis of variance (Duration by Ear) was performed on the number correct for the unmasked identification condition. The analysis revealed a significant effect of duration $[F(1,7)=9.12$, $\mathrm{p}<.025]$; the mean percentage correct for the 22 and 44-msec durations was 78 and 96.

\section{Backward Masking}

Effect of ISI, target duration, ear of presentation. The mean percentage correct. plotted as a function of ISI. is shown for the two target durations in Figure 3a. The analysis of variance (ISI by Duration by Ear) performed on the number correct yielded significant effects due to ISI $[F(6,42)=32.60]$ and duration $[F(1.7)=44.88], p<.001$ in each case. The ear factor was not significant $[F(1,7)=1.86$, $p>.05]$. The mean percentage correct for the 22- and 44-msec durations was 66 and 87 . For the left and right ears, subjects averaged $75 \%$ and $78 \%$ correct.

Effects of phonetic feature composition of target and mask. Evaluation of the six feature contrast predictions for the two target durations showed that differences in accuracy of target identification due to the number of feature contrasts between the target and mask were as predicted in 10 out of $12(83 \%)$ instances. $p<.019$ (see Table 2 ). The selective target feature retention predictions were also evaluated for the two target durations. Overall, the predictions were supported in 11 out of $12(92 \%)$ cases, $p<.001$ (see Table 3). 


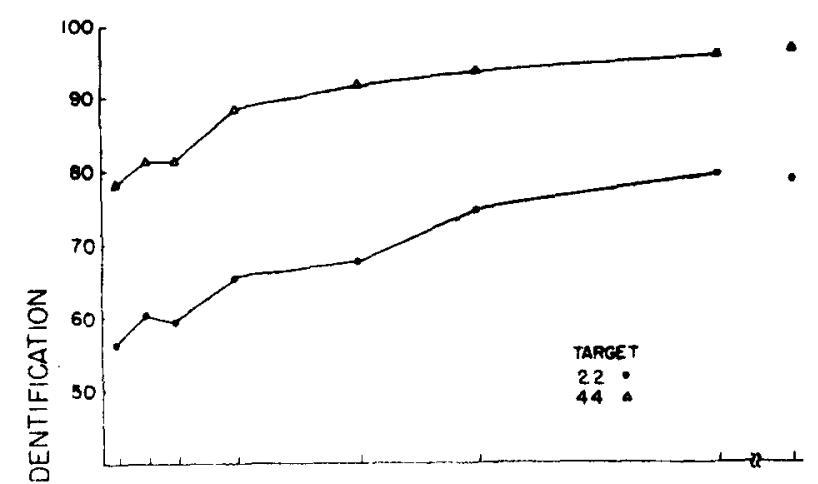

(a)

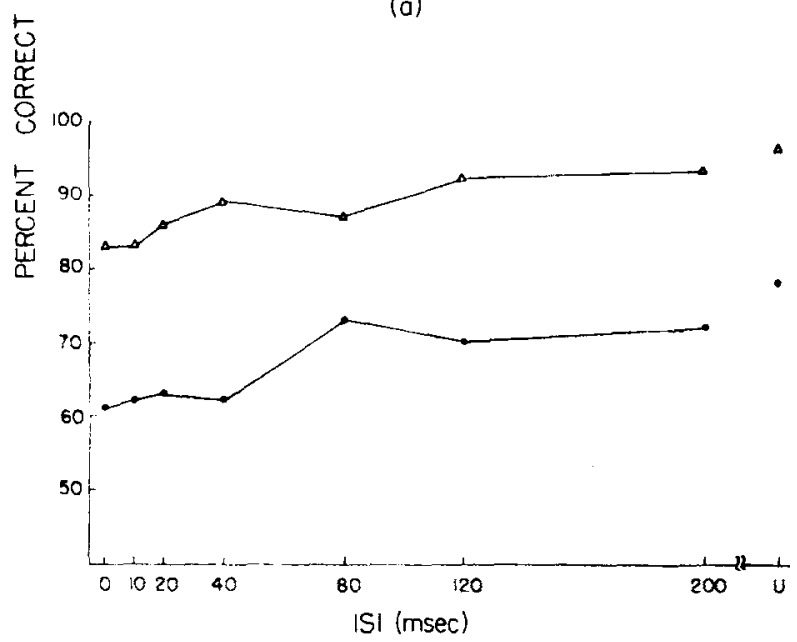

(b)

Figure 3. Mean percent correct identification $(N=8)$ is plotted as a function of ISI for target durations of $22 \mathrm{msec}$ (filled circles) and 44 msec (triangles) for the backward masking condition in (a) and the forward masking condition in (b), Experiment II. The unmasked level of performance for each target duration is indicated at the right by "U."

\section{Forward Masking}

Effects of ISI, target duration, ear of presentation. Figure $3 \mathrm{~b}$ shows the forward masking functions. The analysis of variance found significant effects due to both ISI $[F(6,42)=6.20]$ and duration $[F(1,7)=$ 39.64], at the .001 level in both instances. As in the backward masking condition, the ear factor was not significant $[\mathrm{F}(1,7)=2.98, \mathrm{p}>.05]$. Mean percentage correct for the 22 and 44 msec target durations was 66 and 87 , respectively; for the left and right ears, mean percentage correct was 75 and 78 .

Effects of phonetic feature composition of target and mask. Evaluation of the feature contrast predictions revealed support for the predictions in 10 out of $12(83 \%)$ instances, $p<.019$ (see Table 2). The selective feature retention predictions were supported in 9 of $12(75 \%)$ cases, $p<.073$ (see Table 3).

\section{DISCUSSION OF EXPERIMENTS I AND II}

\section{The Course of Consonant Processing}

In all masking conditions of Experiment I, recognition accuracy increased overall with increments in ISI. In the case of backward masking, this finding suggests that linguistic categorization of consonants is not immediate; the processing leading to phoneme identification continues beyond stimulus termination and may be interfered with by the presentation of another consonant. In the case of forward masking, the results suggest that an early arriving consonant may interfere with the processing of a later arriving consonant. The fact that accuracy in the masked condition was impaired relative to the unmasked condition for all target durations indicates that, although the severity of masking may be affected by target duration, the masking phenomenon is a general one. It is not restricted to a particular target duration or ratio of target-to-mask duration.

The results of Experiment II indicated that the masking observed in Experiment I was not solely a peripheral phenomenon. Masking was observed in both the forward and backward dichotic conditions of Experiment II. Another experiment in this series (see Wolf, 1974, Experiment II) in which the target and mask were presented dichotically, but the target ear remained constant for a block of trials, yielded substantially the same results. Thus, the masking observed in the current experiment did not depend on the subject's uncertainty concerning the target ear.

There were several interesting differences between the dichotic and diotic masking functions. In the. diotic forward masking condition, accuracy was quite low at the shorter ISIs relative to the asymptotic level of performance. The difference between accuracy at the shorter ISIs and the asymptotic level of performance in the dichotic conditions is, by comparison, much smaller. In the case of backward masking, the shapes of the diotic and dichotic 22-msec target duration functions differ over the first $40 \mathrm{msec}$. Whereas accuracy increases monotonically with ISI in the dichotic function, the corresponding diotic function is nonmonotonic; the diotic function reaches a low point at 20-40-msec ISI. The diotic and dichotic backward masking functions also differ in the level of accuracy attained at longer ISIs. By 200-msec ISI, the dichotic backward masking functions have reached the unmasked level of accuracy for each target duration. The diotic functions, in contrast, are well below the unmasked level of performance at 200-msec ISI. Because the diotic presentation conditions of Experiment I allowed for peripheral as well as central target-mask interactions, while the dichotic conditions of Experiment II allowed for central interactions only, 
the differences between the diotic and dichotic masking functions suggest that a target presented under diotic masking conditions may undergo peripheral interterence not experienced by a target presented under dichotic masking conditions.

The magnitude of forward masking found in the present experiments was greater than has typically been reported for nonspeech stimuli (Massaro, 1970; Turvey, 1973). The occurrence of masking in the forward paradigm suggests that the mask is processed in terms of its phonetic features even though it is not to be reported. Although target onset may terminate mask processing with some small delay, phonetic feature analyzers may be engaged in the processing of mask information when target information arrives at the stage of phonetic feature analysis. Target processing may consequently be delayed and some early arriving target information may be lost. Because the targets are characterized by a rapidly changing frequency spectrum, the loss of even a little initial information may seriously alter the characteristics of the target preperceptual auditory image and impair the extraction of target phonetic features. As a result. target features may be lost and previously extracted mask features may replace target features.

An analogous loss of early information may occur for nonspeech steady-state stimuli under forward masking conditions. In the case of steady-state targets, however, the loss of some initial target information may not change the characteristics of the target preperceptual image and so may not interfere with the analysis of target features. Dorman, Kewley-Port. Brady-Wood, and Turvey's (1973) failure to obtain forward masking with steady-state vowel targets indicates that comparable forward masking does not occur for all classes of speech stimuli. and suggests that the transient nature of the critical target information in these experiments may have been responsible for the large forward masking effects.

\section{Phonetic Feature Interactions}

The support observed in the two experiments for the selective target feature retention predictions provides evidence against the hypothesis that the mask impairs target identification by disrupting target information in an all-or-none way. Instead, the findings suggest that the phonetic features of the target may be selectively retained or lost. It may, therefore, be argued that the feature contrast effect observed in both backward and forward masking conditions was due to an interaction of target and mask features.

The fact that the selective target feature retention and feature contrast predictions were supported in the dichotic masking conditions of Experiment II suggests that these effects have a central origin. The somewhat stronger support for these predictions under dichotic masking conditions is, perhaps, attributable to the occurrence of peripheral target-mask interaction in the diotic condition, which was not feature-dependent and which may have obscured central phonetic feature interactions to some extent. Although these feature interactions are assumed to have a phonetic basis, the possibility that the interaction occurs on a central auditory level cannot be ruled out. However, the fact that the feature contrast effect was found for targets and masks of unequal duration (and so with somewhat different auditory characteristics) is evidence that the interaction has a phonetic rather than an auditory basis. ${ }^{4}$

As noted above, Massaro's (1971) backward masking investigations of auditory information processing using tones did not find that the effectiveness of the mask was dependent on target-mask similarity. In a study published after the present experiments were completed. Massaro (1974) made a similar claim for CV targets and masks ([b, d, g] served as both the targets and masks). Inspection of Massaro's (1974) data (Table 2, showing d'values for each target as a function of the mask), however. reveals that two out of three targets were more accurately identified when the target and mask were identical than when they differed.

\section{EXPERIMENT III}

Experiment III assessed the effect of mask duration on recognition accuracy in diotic and dichotic backward masking. In addition to investigating the importance of mask duration. Experiment III was intended to assess the importance of the ratio of target-to-mask duration in backward masking. In Experiment I, greater masking was found for targets which were shorter than the mask than for targets equal in duration to the mask. In Experiment III, mask durations of 22 and $88 \mathrm{msec}$ were used with a target duration of $22 \mathrm{msec}$. Thus, it was possible to assess the effects of the ratio of target-to-mask duration on accuracy of identification using a target of constant duration for which large masking effects had previously been found. A secondary purpose of Experiment III was to assess the effects of diotic vs. dichotic target-mask presentation within the same group of subjects.

\section{Method}

\section{Stimuli, Test Orders, Apparatus}

The unmasked and masked identification tapes for Experiment III were identical to those of Experiment II, with the exception that a target duration of $22 \mathrm{msec}$ was used in all masked identification test orders and mask durations of 22 and $88 \mathrm{msec}$ were employed in separate test orders.

For diotic target-mask presentation, the outputs from the two channels of the tape recorder were mixed as in Experiment I; for dichotic presentation, the apparatus was arranged as in 
Experiment II, so that the outputs from the two channels of the tape recorder went to different ears. Thus, in the dichotic condition, the target ear varied randomly from trial to trial.

\section{Subject}

Eight students from Providence, R.I., area colleges served as subjects. All subjects were native speakers of English, right-handed, and had no known hearing impairment in either ear.

\section{Design and Procedure}

The experiment was conducted in three 90 -min sessions. The unmasked identification test orders (diotic and monotic) were presented before and after the masked identification test orders, as in the other experiments. In order to familiarize subjects with the synthetic stimuli, unmasked identification test orders with the 44-msec-duration stimuli were presented diotically or monotically before the corresponding 22-msec test order given at the start of the session. Within each session, all masked identification test orders of one presentation condition (diotic or dichotic) were given before the test orders of the other condition. The order of presentation of the diotic and dichotic test orders were counterbalanced across subjects and sessions. Other design and procedural details were as described for Experiment II.

\section{Results \\ Unmasked Identification}

An analysis of variance for the factor of target ear (left, right, or both) revealed that factor to have statistically significant effects $[F(2,14)=11.78$, $\mathrm{p}<.001]$. Evaluation of the ear effect indicated that accuracy for targets presented diotically $(88 \%$ correct) was significantly greater than accuracy for targets presented to one ear $(74 \%$ correct $), p<.01$.

\section{Diotic and Dichotic Backward Masking}

Effects of ISI, mask duration, target ear. The mean percentage correct is plotted as a function of ISI for the two mask durations for the diotic and dichotic conditions in Figures $4 a$ and $4 b$, respectively. The data from the diotic and dichotic conditions were analyzed together in an analysis of variance for ISI by Mask Duration by Target Ear. The analysis revealed significant effects due to ISI $[F(6,42)=12.25]$ and mask duration $[F(1,7)=30.99], \mathrm{p}<.001$ or better in both cases. Subjects averaged $55 \%$ correct with the 88 -msec mask and $66 \%$ correct with the 22 -msec mask. The ear factor was marginally significant $[F(2,14)=3.69, p<.10]$. Because the comparisons of interest for the ear factor were diotic vs. dichotic performance and left- vs. right-ear performance, these comparisons were made using Lindquist's critical difference. The analyses revealed that right-ear performance $(63 \%$ correct) was superior to left-ear performance $(56 \%$ correct), $\mathrm{p}<.05$.

Effect of phonetic feature composition of target and mask. The feature contrast and selective target feature retention predictions were evaluated for the 22- and 88-msec mask durations for the diotic and dichotic conditions. The feature contrast predictions were supported in 10 out of 12 instances in both the dichotic and diotic conditions, $\mathrm{p}<.019$ (see Table 2 ). The selective feature retention predictions were

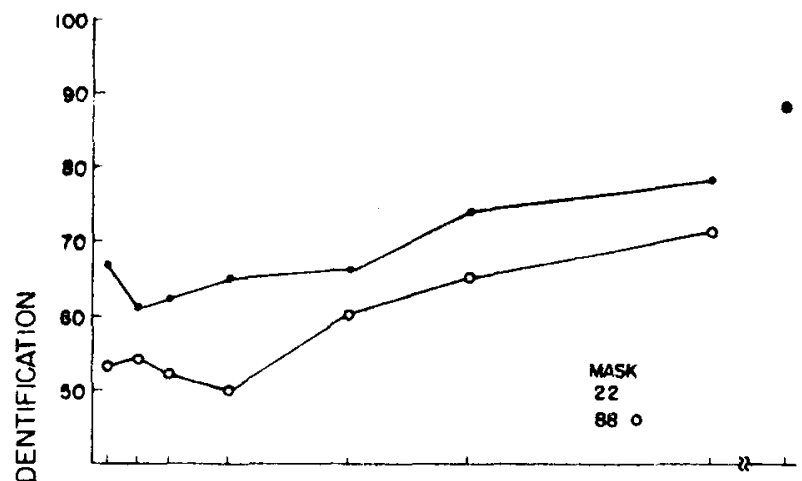

(a)

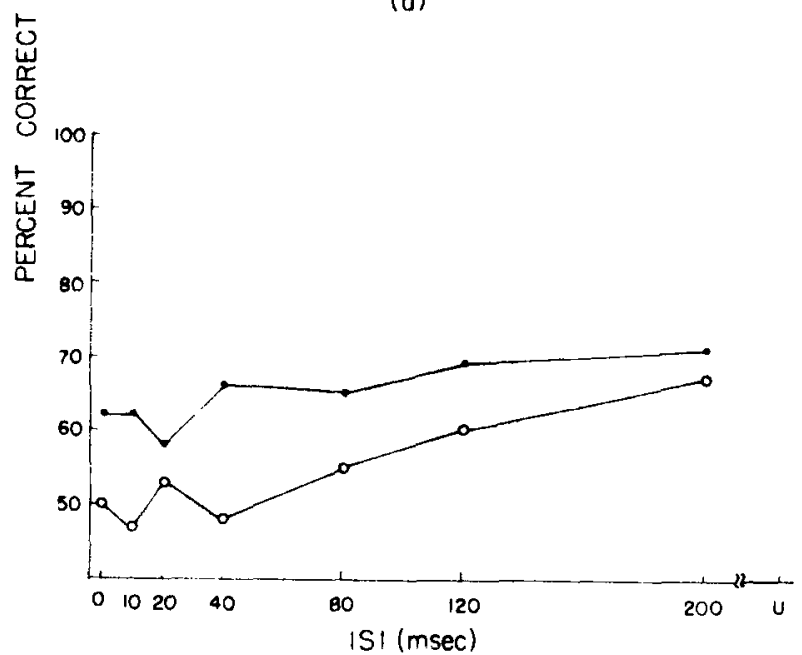

(b)

Figure 4. Mean percent correct identification $(N=8)$ plotted as a function of ISI for mask duration of 22 msec (filled circles) and 88 msec (open cheles) for the diotic mauling condition in (a) and the dichotic condition in (b), Experiment III. The unmasked level of performance is indicated at the right by "U."

supported in 9 out of $12(75 \%)$ instances for the dichotic conditions, $p<.073$. In the diotic conditions, however, only 4 out of $12(33 \%)$ predictions were supported, $\mathrm{p}>.50$ (see Table 3).

\section{Mask Duration}

\section{Discussion}

Experiment III clearly demonstrated that substantial backward masking may occur with a mask and target $\mathrm{CV}$ of equal duration under both diotic and dichotic conditions. The results, however, also indicated that masking was more severe with the 88-msec mask than with the 22-msec mask in both dichotic and diotic conditions. Moreover, the reduction in accuracy of target identification attributable to an increase in mask duration did not differ in the diotic and dichotic conditions. This finding leads to the conclusion that variations in mask duration affect central processes only. One possible explanation for the central nature of the mask duration effect is that phonetic feature information is 
more readily extracted from the longer masks than from the shorter masks, due either to the inherently better quality of the phonetic information in the longer masks, or to the lesser interfering effect of the target on the characteristics of the preperceptual auditory image of the longer masks. As a result, the longer masks compete more effectively with the targets for the phonetic feature analyzers and thus cause more interference with the extraction of target features.

It is also possible that an increase in mask duration lowered accuracy by lowering the ratio of target-to-mask energy. Using the standard recognition masking paradigm and $\mathrm{CV}$ targets and masks, Pisoni (1972), however, found that manipulations of target-mask energy relations made by varying mask intensity had essentially no effect on accuracy of consonant identification under dichotic backward masking conditions. ${ }^{5}$ Moreover, the fact that Massaro (1971) found no effect of mask duration on accuracy of target identification for tonal stimuli under diotic backward masking conditions argues for an explanation of the mask duration effect in terms of the phonetic (or auditory) characteristics of these stimuli.

\section{Diotic and Dichotic Masking}

A comparison of identification accuracy under diotic and dichotic conditions substantiates the proposal that a target presented under diotic masking conditions may undergo transmission line interference not experienced by a target presented alone or under dichotic masking conditions. Although accuracy was greater for stimuli presented diotically than for stimuli presented to one ear in the unmasked identification task, the difference between diotic and dichotic conditions was not significant in the masking task. In other words, relative to unmasked identification performance, the mask lowered accuracy somewhat more in the diotic conditions than in the dichotic conditions. As was true for Experiments I and II, the level of performance attained at the longest ISI was closer to the unmasked level in the dichotic than in the diotic condition. It is likely that these findings reflect the operation of two distinct underlying processes with opposite effects. Diotic presentation may provide redundant information by the presentation of the target to both ears. In the case of very brief stimuli, such as those employed in the present experiments, this redundancy may be useful in target identification. Consequently, performance in the unmasked identification task was better for diotic than for dichotic (actually, monotic) target presentation. Under masking conditions, however, diotic target-mask presentation may cause transmission line interference between the target and mask, in addition to the facilitation due to redundant presentation of the target to the two ears. Thus, under diotic masking conditions, the effects of transmission line interference may cancel the effects of target redundancy.

Contrary to the present findings, Pisoni (1972) found dichotic backward masking to be more severe than diotic masking. The difference between Pisoni's results and those of the present experiment may be due to the use of an identical target and mask set in Pisoni's (1972) study; the poorer performance under dichotic presentation observed in that study may have been due to a larger number of temporal order judgment errors in the dichotic than in the diotic condition.

It is interesting to note that the $88 \mathrm{msec}$ mask duration diotic function of Experiment III (Figure $4 a$ ) is quite similar in shape to the corresponding backward masking function of Experiment I (Figure 2a). One possible explanation for these $\mathrm{U}$-shaped functions is that, due to the sequential nature of auditory stimuli, the auditory information processing system may tend to treat all information received over the same channel (i.e., ear) within a particular time span as one signal. As a consequence, a very brief target and the following mask may be processed as one unit at short ISIs. Thus, at short ISIs, the target may, to some extent, escape the peripheral interference caused by the mask. Since the U-shaped functions were found only with the $22-\mathrm{msec}$ target and 88-msec mask, the phenomenon appears to depend on both target and mask duration, or perhaps the ratio of target-to-mask duration. This proposal is obviously speculative and awaits confirmation or disconfirmation by further experimentation.

The predictions based on the selective retention of target features received support in the dichotic condition, but not in the diotic condition. There is no obvious explanation for the difference between the findings concerning the selective target feature retention predictions for the diotic condition of Experiment III and corresponding condition of Experiment 1.

\section{GENERAL DISCUSSION}

The findings of these experiments indicate that phonetic categorization of consonants is not immediate; the processes leading to consonant identification appear to continue beyond the termination of a brief $\mathrm{CV}$ stimulus. When a mask $\mathrm{CV}$ is presented after a target $\mathrm{CV}$, as in backward masking. ongoing target processing is interfered with by the mask. When a target $\mathrm{CV}$ is presented after a mask $\mathrm{CV}$, as in forward masking, ongoing mask processing interferes with target processing. The experiments also suggest that consonant masking may occur at at least two loci: peripherally, prior to the establishment of the preperceptual auditory image, and centrally, subsequent to the establishment of the 
preperceptual auditory image, but prior to phoneme identification.

Although the results of statistical tests of the selective target feature retention and feature contrast predictions did not reach conventional levels of significance in all cases, taken together, the evidence strongly suggests that central masking involves an interaction of target and mask features. Across all conditions of the three experiments, the selective target feature retention predictions were supported in $73 \%$ of the cases. For the $44-\mathrm{msec}$ targets, the stimuli which most closely approximated normal speech in terms of the duration of a number of important consonant cues, the predictions were supported $92 \%$ of the time. The feature contrast predictions were supported in $77 \%$ of the cases overall. Nearly half $(47 \%)$ of the cases in which the feature contrast predictions were not supported involved the same prediction (Prediction 2, Table 2).

It is suggested that the target-mask phonetic feature interaction occurs during phonetic feature extraction, prior to phoneme identification. This interaction may arise as follows. Phonetic feature analyzing mechanisms may operate independently of each other; certain features may be more rapidly or accurately extracted than others. Consequently, at any point in the processing of a consonant, information may be available for some phonetic features but not for others. In backward masking, target phonetic feature information may not be completely extracted when the mask is presented. Because the same set of phonetic features are relevant to the identification of target and mask set members, the mask and target may compete for the same phonetic feature analyzers. As a result, the mask interferes with the extraction of target phonetic feature information, and target feature information not yet extracted at the time of mask presentation may be lost. The lost target information may in some instances be replaced by feature information from the mask. In forward masking, target phonetic feature information may be lost if ongoing mask processing delays target processing. If a target feature has been lost and a mask feature has replaced the lost target information, the phoneme reported will reflect that mask feature. If a lost target feature has not been replaced by a mask feature, identification will be based on the incomplete set of features.

It should be pointed out that central interactions are not by necessity limited to those occurring at the level of phonetic features. Central interactions of a strictly auditory nature may occur prior to phonetic feature extraction. It is possible that the considerable number of errors in dichotic masking which cannot be attributed to phonetic feature interactions were due to central auditory interactions.

\section{Final Comment}

The present experiments have demonstrated the importance of parallel investigations of speech and nonspeech information processing. The use of a paradigm such as recognition masking in the investigation of speech as well as nonspeech reveals the similarities and differences between the processing of speech and nonspeech and contributes to the formulation of a general theory of auditory information processing.

\section{REFERENCE NOTE}

1. Pisoni, D. B. Perceptual processing time for consonants and vowels. Paper presented at the meeting of the Acoustical Society of America, December 1972.

\section{REFERENCES}

DARwIN, C. J. Dichotic backward masking of complex sounds. Haskins Labonatories Status Report on Speech Research, 1971, 27, 199-207.

Dorman, M., Kewley-Port, D., Brady-Wood, S., \& TuRvey, M. Forward and backward masking of brief vowels. Haskins Laboratories Status Report on Speech Research, 1973, 33, 93-100.

Liberman, A. M. The grammars of speech and language. Cognitive Psychology, 1970, 1, 201-301.

Lindpuist, E. Design and analysis of experiments in psychology and education. Boston: Houghton Mifflin, 1953.

Lisker, L., \& ABramson, A. S. The voicing dimension: Some experiments in comparative phonetics. In Proceedings of the Sixth International Congress of Phonetic Sciences, 1967. Prague: Academia, 1970. Pp. 563-567.

MAssaro, D. W. Preperceptual auditory images. Joumal of Experimental Psychology, 1970, 85, 411-417.

MASSARO, D. W. Effect of masking tone duration on preperceptual auditory images. Journal of Experimental Psychology, 1971, 87, 146-148.

Massaro, D. W. Preperceptual images, processing time, and perceptual units in auditory perception. Psychological Review, 1972. 79, 124-145. (a)

Massaro, D. W. Stimulus information vs. processing time in auditory pattern recognition. Perception \& Psychophysics, $1972,12,50-56$. (b)

MAssaro, D. W. A comparison of forward versus backward recognition masking. Joumal of Experimental Psychology, $1973,100,434-436$.

Massaro, D. W. Perceptual units in speech recognition. Journal of Experimental Psychology, 1974, 102, 199-208.

McNeill, D., \& RePP, B. Internal processes in speech perception. Joumal of the Acoustical Society of America, 1973, 53, 1320-1326.

Morton, J.. \& Brondbent, D. E. Passive versus active recognition models or is your homunculus really necessary? In W. Wathen-Dunn (Ed.), Models for the perception of speech and visual form. Cambridge, Mass: M.I.T. Press, 1967. Pp. 103-110.

Pison, D. B., \& McNarb. S. D. Dichotic interactions and phonetic feature processing. Brain and Language, 1974, 1. 351-362.

REPP, B. H. Dichotic forward and backward "masking" between CV syllables. Joumal of the Acoustical Society of America, 1975, 57, 483-496. 
Stevens, K. N.. \& Halle. M. Remarks on analusis by synthesis and distinctive features. In W. Wathen-Dunn (Ed.). Models for the perception of speech and visual form. Cambridge, Mass: M.I.T. Press, 1967. Pp. 88-102.

Stevens. K. N., \& House, A. S. Speech perception. In J. Tobias (Ed.). Foundations of modern auditon theory (Vol. 2). New York: Academic Press. 1972. Pp. 3-62.

STUDDERT-KenNEDY. M. The perception of speech. Haskins Laboratories Status Report on Speech Research. 1970. 21/22. $15-48$.

Silddert-Kennedy, M.. Shankweiler. D.. \& Schulman, S. Opposed effects of a delayed channel on perception of dichotically and monotically presented $\mathrm{CV}$ syllables. Journal of the Acoustical Socien of America. 1970. 48. 599-602.

TuRveY. M. T. On peripheral and central processes in vision: Inferences from an information-processing analysis of masking with patterned stimuli. Psychological Revieu, 1973. 80. 1-52.

WOLF. C. G. An analysis of speech processing: Some implications from studies of recognition masking. Unpublished $\mathrm{PhD}$ thesis. Brown University. 1974.

\section{NOTES}

1. In monotic presentation. one or more stimuli are presented to the same ear. whereas in dichotic presentation. two different stimuli are presented, one to each ear. Diotic presentation involves the presentation of the same stimulus or stimuli to both ears.

2. It is also possible that the feature-sharing effect reported by Pisoni and McNabb (1974) and McNeill and Repp (1973) was due to a mask-produced response bias. For example. subjects may have a greater tendency to report [d] for the mask [b] than for the mask [p] regardless of the target. Consequently. accuracy of target identification will be greater for the target-mask pair [d]-[b]. differing by one feature, than the pair. [d]-[p]. differing by two features. I am grateful to Dr. Dominic Massaro for making this point with reference to $\mathrm{my}$ own data.

3. It should be noted that since the target and mask sets consist of different stimuli. feature replacement may result in a matrix of features appropriate to a mask set member. For example. when the voicing value of the target $[d]$ is replaced by the voicing value of the mask [p], a set of features appropriate to the mask [t] results. It is assumed. for simplicity. that subjects guess randomly among the three targets in such cases. These same predictions are obtained. however. if it is assumed that subjects guess selectively between those targets which differ from the perceived matrix of features by one feature (see Wolf. 1974, for details).

4. It would. of course, be of interest to assess the feature contrast and selective target feature retention hypotheses as a function of ISI. Because there were not enough errors at each ISI to make such an analysis feasible. it cannot be determined from the present data whether the feature contrast and selective target feature retention effects extend throughout the entire range of ISIs.

5. Pisoni (1972) does not discuss the effects of mask intensity in his paper. This observation is made on the basis of inspection of his Figure 5.

(Received for publication April 30. 1975: revision accepted September 10.1975.) 